J. Korean Math. Soc. 52 (2015), No. 1, pp. 141-157

http://dx.doi.org/10.4134/JKMS.2015.52.1.141

\title{
ADMIXABLE OPERATORS AND A TRANSFORM SEMIGROUP ON ABSTRACT WIENER SPACE
}

\author{
Seung Jun Chang, Jae Gil Choi, and David Skoug
}

\begin{abstract}
The purpose of this paper is first of all to investigate the behavior of admixable operators on the product of abstract Wiener spaces and secondly to examine transform semigroups which consist of admixWiener transforms on abstract Wiener spaces.
\end{abstract}

\section{Introduction}

Let $C_{0}[0, T]$ denote one-parameter Wiener space, that is the space of all real-valued continuous functions $x$ on $[0, T]$ with $x(0)=0$. Let $\mathcal{M}$ denote the class of all Wiener measurable subsets of $C_{0}[0, T]$ and let $m_{\mathrm{w}}$ denote Wiener measure. Then $\left(C_{0}[0, T], \mathcal{M}, m_{\mathrm{w}}\right)$ is a complete measure space.

In [1], Bearman presented a seminal result for Wiener integral on product Wiener space. It can be summarized as follows:

Theorem 1.1. Let $\mathbf{F}$ be a $m_{\mathrm{w}} \times m_{\mathrm{w}}$-integrable functional on $C_{0}^{2}[0, T]$, the product of 2 copies of the Wiener space $C_{0}[0, T]$, and let $\theta$ be a function of bounded variation on $[0, T]$. Let $T_{\theta}: C_{0}^{2}[0, T] \rightarrow C_{0}^{2}[0, T]$ be the transformation defined by $T_{\theta}(w, z)=(x, y)$ with

$$
\left\{\begin{array}{c}
x(t)=\int_{0}^{t} \cos \theta(s) d w(s)-\int_{0}^{t} \sin \theta(s) d z(s), \\
y(t)=\int_{0}^{t} \sin \theta(s) d w(s)+\int_{0}^{t} \cos \theta(s) d z(s) .
\end{array}\right.
$$

Then the transform $T_{\theta}$ is measure preserving and

$$
\int_{C_{0}^{2}[0, T]} \mathbf{F}\left(T_{\theta}(w, z)\right) d\left(m_{\mathrm{w}} \times m_{\mathrm{w}}\right)(w, z)=\int_{C_{0}^{2}[0, T]} \mathbf{F}(x, y) d\left(m_{\mathrm{w}} \times m_{\mathrm{w}}\right)(x, y) .
$$

As a special case of (1.2), one can see that for any Wiener integrable functional $F$ on $C_{0}[0, T]$ and all $\theta \in \mathbb{R}$,

$$
\int_{C_{0}^{2}[0, T]} F(w \cos \theta+z \sin \theta) d\left(m_{\mathrm{w}} \times m_{\mathrm{w}}\right)(w, z)=\int_{C_{0}[0, T]} F(x) d m_{\mathrm{w}}(x) .
$$

Received April 14, 2014.

2010 Mathematics Subject Classification. 28C20, 46G12, 20M20.

Key words and phrases. abstract Wiener space, admixable operator, admix-Wiener transform, transform semigroup. 
In view of equation (1.2), we see that the Wiener measure $m_{\mathrm{w}} \times m_{\mathrm{w}}$ is rotation invariant. Equation (1.3) has played an important role in various research areas in mathematics and physics involving Wiener integration theory. Equation (1.3) with the change of scale of Wiener measure was further developed by Cameron and Storvick [2] and by Johnson and Skoug [10] in their studies of Wiener integral equations. In [14], Lee extended equation (1.3) on the complexification of abstract Wiener space to study the solutions of the differential equation which is called a Cauchy problem.

The results obtained in this paper are established in three sections. In Section 3 we obtain a rotation property for admixable operators on the product of abstract Wiener spaces. In Section 4 we apply our rotation property from Section 3 to prove Bearman's rotation property, namely Theorem 1.1 above. In Section 5 we define an admix-Wiener transform on abstract Wiener space and proceed to show that the admix-Wiener transforms form a transform semigroup on abstract Wiener space.

\section{Preliminaries}

Let $H$ be a real separable infinite-dimensional Hilbert space with inner product $\langle\cdot, \cdot\rangle$ and norm $|\cdot|=\sqrt{\langle\cdot, \cdot\rangle}$. Let $\|\cdot\|_{0}$ be a measurable norm on $H$ with respect to the Gaussian cylinder set measure $\nu_{0}$ on $H$. Let $B$ denote the completion of $H$ with respect to $\|\cdot\|_{0}$. Let $i$ denote the natural injection from $H$ to $B$. The adjoint operator $i^{*}$ of $i$ is one to one and maps $B^{*}$ continuously onto a dense subset of $H^{*}$, where $B^{*}$ and $H^{*}$ are topological duals of $B$ and $H$, respectively. By identifying $H^{*}$ with $H$, and $B^{*}$ with $i^{*} B^{*}$, we have a triple $B^{*} \subset H^{*} \approx H \subset B$ and $\langle x, y\rangle=(x, y)$ for all $x$ in $H$ and $y$ in $B^{*}$, where $(\cdot, \cdot)$ denotes the natural dual pairing between $B$ and $B^{*}$. By the well-known result of Gross [7], $\nu_{0} \circ i^{-1}$ provides a unique countably additive extension, $\nu$, to the Borel $\sigma$-algebra $\mathcal{B}(B)$ of $B . \nu$ is a probability measure on the Borel $\sigma$-algebra $\mathcal{B}(B)$ of $B$ which satisfies

$$
\int_{B} \exp \{i(y, x)\} d \nu(x)=\exp \left\{-\frac{1}{2}|y|^{2}\right\} \text { for every } y \in B^{*} .
$$

The triple $(B, H, \nu)$ is called an abstract Wiener space. For more details, see $[4,7,12,13]$.

Let $\left\{e_{j}\right\}_{j=1}^{\infty}$ be a complete orthonormal set in $H$ such that the $e_{j}$ 's are in $B^{*}$. For each $h \in H$ and $x \in B$, we define the stochastic inner product $(h, x)^{\sim}$ by

$$
(h, x)^{\sim}=\left\{\begin{array}{cl}
\lim _{n \rightarrow \infty} \sum_{j=1}^{n}\left\langle h, e_{j}\right\rangle\left(e_{j}, x\right), & \text { if the limit exists, } \\
0, & \text { otherwise. }
\end{array}\right.
$$

For every $h(\neq 0)$ in $H,(h, x)^{\sim}$ exists for $\nu$-a.e. $x \in B$ and it is a Gaussian random variable on $B$ with mean zero and variance $|h|^{2}$, i.e., equation (2.1) holds with $y \in B^{*}$ replaced with $h \in H$. In fact, the stochastic inner product $(h, x)^{\sim}$ is essentially independent of the choice of the complete orthonormal set 
used in its definition. Also, if both $h$ and $x$ are in $H$, then Parseval's identity gives $(h, x)^{\sim}=\langle h, x\rangle$. Furthermore, $(h, \lambda x)^{\sim}=(\lambda h, x)^{\sim}=\lambda(h, x)^{\sim}$ for any $\lambda \in \mathbb{R}, h \in H$ and $x \in B$. We also see that if $\left\{h_{1}, \ldots, h_{n}\right\}$ is an orthogonal set in $H$, then the random variables $\left(h_{j}, x\right)^{\sim}$ 's are independent.

Next we state the definition of the admixable operator on $B$. For more details, see [3].

Definition 2.1. Let $\odot$ be an operation between $H$ and $B^{*}$, which satisfies the conditions:

(a1) $B^{*} \times B^{*} \ni\left(g_{1}, g_{2}\right) \mapsto g_{1} \odot g_{2}=g_{2} \odot g_{1} \in B^{*}$.

(a2) $H \times B^{*} \ni(h, g) \mapsto h \odot g=g \odot h \in H$.

(a3) For every $h \in H$ and every $g_{1}, g_{2} \in B^{*}$,

$$
\left(h \odot g_{1}\right) \odot g_{2}=h \odot\left(g_{1} \odot g_{2}\right) .
$$

(a4) For every $h_{1}, h_{2} \in H$ and every $g \in B^{*}$,

$$
\left(h_{1}+h_{2}\right) \odot g=h_{1} \odot g+h_{2} \odot g .
$$

(a5) For every $g_{1}, g_{2} \in B^{*}$, there exists $g_{3} \in B^{*}$ such that

$$
g_{1}^{\odot 2}+g_{2}^{\odot 2}=g_{3}^{\odot 2},
$$

where $g^{\odot 2}=g \odot g$. In this case, we write

$$
g_{3} \equiv \sqrt[\odot]{g_{1}^{\odot 2}+g_{2}^{\odot 2}} \equiv s\left(g_{1}, g_{2}\right)
$$

(a6) For every $h_{1}, h_{2} \in H$ and every $g \in B^{*}$,

$$
\left\langle h_{1}, h_{2} \odot g\right\rangle=\left\langle h_{1} \odot g, h_{2}\right\rangle .
$$

Given $g \in B^{*}$, let $A_{g}: B \rightarrow B$ be a linear operator associated with $g$. The operator $A_{g}$ is said to be $g^{\odot}$-admixable, provided $\left(h, A_{g} x\right)^{\sim}=(h \odot g, x)^{\sim}$ for all $h \in H$.

For a finite subset $\mathcal{V}=\left\{v_{1}, \ldots, v_{m}\right\}$ of $H$, let $X_{\mathcal{V}}: B \rightarrow \mathbb{R}^{m}$ denote the random vector given by

$$
X_{\mathcal{V}}(x) \equiv\left(\left(v_{1}, x\right)^{\sim}, \ldots,\left(v_{m}, x\right)^{\sim}\right) .
$$

A functional $F$ is called a cylinder-type functional on $B$ if there exists a linearly independent subset $\mathcal{V}=\left\{v_{1}, \ldots, v_{m}\right\}$ of $H$ such that

$$
F(x)=\psi\left(\left(v_{1}, x\right)^{\sim}, \ldots,\left(v_{m}, x\right)^{\sim}\right) \equiv \psi\left(X_{\mathcal{V}}(x)\right), \quad x \in B,
$$

where $\psi$ is a complex-valued Borel measurable function on $\mathbb{R}^{m}$. It is easy to show that for the cylinder-type functional $F$ of the form (2.5), there exists an orthogonal subset $\mathcal{H}=\left\{h_{1}, \ldots, h_{n}\right\}$ of $H$ such that $F$ is expressed as

$$
F(x)=f\left(\left(h_{1}, x\right)^{\sim}, \ldots,\left(h_{n}, x\right)^{\sim}\right) \equiv f\left(X_{\mathcal{H}}(x)\right), \quad x \in B,
$$

where $f$ is a complex-valued Borel measurable function on $\mathbb{R}^{n}$. Thus, we loose no generality in assuming that every cylinder-type functional on $B$ is of the form (2.6). 
For $g \in B^{*}$, let $A_{g}$ be the $g^{\odot}$-admixable operator on $B$ and let $F$ be given by equation (2.6). Then

$$
\begin{aligned}
F\left(A_{g} x\right) & =f\left(\left(h_{1}, A_{g} x\right)^{\sim}, \ldots,\left(h_{n}, A_{g} x\right)^{\sim}\right) \\
& =f\left(\left(h_{1} \odot g, x\right)^{\sim}, \ldots,\left(h_{n} \odot g, x\right)^{\sim}\right) .
\end{aligned}
$$

Even though the subset $\mathcal{H}=\left\{h_{1}, \ldots, h_{n}\right\}$ of $H$ is orthogonal, the subset $\mathcal{H} \odot g \equiv$ $\{h \odot g: h \in \mathcal{H}\}$ of $H$ need not be orthogonal. However, applying the GramSchmidt procedure, we can find an orthogonal subset $\left\{k_{1}, \ldots, k_{m}\right\}$ ( $m$ may be less than $n$ ) and a Borel measurable function $f^{*}: \mathbb{R}^{m} \rightarrow \mathbb{C}$ such that

$$
F\left(A_{g} x\right)=f^{*}\left(\left(k_{1}, x\right)^{\sim}, \ldots,\left(k_{m}, x\right)^{\sim}\right) .
$$

In view of these observations, we shall always choose $g \in B^{*}$ for the admixable operator $A_{g}$, so that $\mathcal{H} \odot g$ is orthogonal in $H$.

Let $\mathcal{H}=\left\{h_{1}, \ldots, h_{n}\right\}$ be an orthogonal subset of $H$ which has no zero and let $\mathcal{O}(\mathcal{H})$ be the class of all elements $g \in B^{*}$ such that $\mathcal{H} \odot g$ is orthogonal in $H$. Because $\operatorname{dim} H=\infty$, infinitely many elements, $g$, exist in $\mathcal{O}(\mathcal{H})$.

Example 2.2. For any orthogonal subset $\mathcal{H}=\left\{h_{1}, \ldots, h_{n}\right\}$ of $H$, each of whose element is a nonzero element of $B^{*}$, let $L(S)$ be the subspace of $H$ which is spanned by the set $S=\left\{h_{i} \odot h_{j}: 1 \leq i<j \leq n\right\}$, and let $L(S)^{\perp}$ be the orthogonal complement of $L(S)$. Let

$$
\mathcal{P}(\mathcal{H})=\left\{g \in B^{*}: g^{\odot 2} \in L(S)^{\perp} \text { and }|g|>0\right\} .
$$

Since $\operatorname{dim} L(S)$ is finite and $B^{*}$ is dense in $H, \operatorname{dim}\left(L(S)^{\perp} \cap B^{*}\right)=\infty$ and so $\mathcal{P}(\mathcal{H})$ has infinitely many elements.

Let $g$ be an element of $\mathcal{P}(\mathcal{H})$. Without loss of generality, we may assume that $\left|h_{j} \odot g\right|>0$ for all $j \in\{1, \ldots, n\}$. By the conditions of the operation $\odot$ and the definition of the $\mathcal{P}(\mathcal{H})$, it follows that for $i, j \in\{1, \ldots, n\}$ with $i \neq j$,

$$
\begin{aligned}
\left\langle h_{i} \odot g, h_{j} \odot g\right\rangle & =\left\langle h_{i} \odot g, g \odot h_{j}\right\rangle=\left\langle h_{i} \odot g \odot h_{j}, g\right\rangle \\
& =\left\langle h_{i} \odot h_{j} \odot g, g\right\rangle=\left\langle h_{i} \odot h_{j}, g \odot g\right\rangle \\
& =\left\langle h_{i} \odot h_{j}, g^{\odot 2}\right\rangle=0 .
\end{aligned}
$$

From this, we see that $\mathcal{H} \odot g \equiv\left\{h_{1} \odot g, \ldots, h_{n} \odot g\right\}$ is an orthogonal subset of $H$ for any $g$ in $\mathcal{P}(\mathcal{H})$, i.e., $\mathcal{P}(\mathcal{H}) \subset \mathcal{O}(\mathcal{H})$.

The following two lemmas will be very useful in the next section where we establish a rotation property for Wiener integrals using admixable operators.

Lemma 2.3. Let $(B, H, \nu)$ be an abstract Wiener space. Let $\mathcal{A}=\left\{\alpha_{1}, \ldots, \alpha_{n}\right\}$ be an orthogonal set in $H$, let $X_{\mathcal{A}}$ be given by (2.4) with $\mathcal{V}$ replaced with $\mathcal{A}$ and 
let $f: \mathbb{R}^{n} \rightarrow \mathbb{C}$ be a Borel measurable function. Then

$(2.7)$

$$
\begin{aligned}
& \int_{B} f\left(X_{\mathcal{A}}(x)\right) d \nu(x) \equiv \int_{B} f\left(\left(\alpha_{1}, x\right)^{\sim}, \ldots,\left(\alpha_{n}, x\right)^{\sim}\right) d \nu(x) \\
\stackrel{*}{=} & \left(\prod_{j=1}^{n} 2 \pi\left|\alpha_{j}\right|^{2}\right)^{-1 / 2} \int_{\mathbb{R}^{n}} f\left(u_{1}, \ldots, u_{n}\right) \exp \left\{-\sum_{j=1}^{n} \frac{u_{j}^{2}}{2\left|\alpha_{j}\right|^{2}}\right\} d u_{1} \cdots d u_{n},
\end{aligned}
$$

where by $\stackrel{*}{=}$ we mean that if either side exists, both sides exist and equality holds.

Lemma 2.4. Let $(B, H, \nu)$ be an abstract Wiener space. Let $R: B \times B \rightarrow$ $\mathbb{R}^{m}$ be a $\mathbb{R}^{m}$-valued Gaussian random vector. Then for any Borel measurable function $f: \mathbb{R}^{m} \times \mathbb{R}^{m} \rightarrow \mathbb{C}$,

$$
\begin{aligned}
& \int_{B \times B} f(R(w, z)) d(\nu \times \nu)(w, z) \\
\stackrel{*}{=} & \left((2 \pi)^{m} \operatorname{det} V\right)^{-1 / 2} \int_{\mathbb{R}^{m}} f(\vec{u}) \exp \left\{-\frac{1}{2} \vec{u} V^{-1} \vec{u}^{T}\right\} d \vec{u},
\end{aligned}
$$

where $V$ is the covariance matrix of the random vector $R$ and $\vec{u}^{T}$ denotes the transpose of the vector $\vec{u}=\left(u_{1}, \ldots, u_{m}\right) \in \mathbb{R}^{m}$.

In order to simplify various expressions in our results below, we adopt the following notations:

$$
f(\vec{u}) \equiv f\left(u_{1}, \ldots, u_{n}\right)
$$

and

$$
f\left(\vec{u}+X_{\mathcal{A}}(x)\right) \equiv f\left(u_{1}+\left(\alpha_{1}, x\right)^{\sim}, \ldots, u_{n}+\left(\alpha_{n}, x\right)^{\sim}\right)
$$

for $\vec{u}=\left(u_{1}, \ldots, u_{n}\right) \in \mathbb{R}^{n}$ and $\mathcal{A}=\left\{\alpha_{1}, \ldots, \alpha_{n}\right\} \subset H$.

\section{A rotation via admixable operator}

Given an orthogonal set $\mathcal{H}$ in $H$, let $\mathfrak{G}_{\mathcal{H}}\left(B^{2}\right)$ be the class of all $\nu \times \nu$ integrable functionals, $\mathbf{F}: B \times B \rightarrow \mathbb{C}$, given by

$$
\begin{aligned}
\mathbf{F}(x, y) & =f\left(\left(h_{1}, x\right)^{\sim}, \ldots,\left(h_{n}, x\right)^{\sim} ;\left(h_{1}, y\right)^{\sim}, \ldots,\left(h_{n}, y\right)^{\sim}\right) \\
& \equiv f\left(X_{\mathcal{H}}(x) ; X_{\mathcal{H}}(y)\right)
\end{aligned}
$$

for $\nu \times \nu$-a.e. $(x, y) \in B \times B$, where $f: \mathbb{R}^{2 n} \rightarrow \mathbb{C}$ is a Borel measurable function.

The following theorem plays a key role in the proof of Theorem 1.1 above.

Theorem 3.1. Let $(B, H, \nu)$ be an abstract Wiener space. Let $\mathcal{H}=\left\{h_{1}, \ldots, h_{n}\right\}$ be an orthogonal subset of $H$ which has no zero, and let $\mathbf{F} \in \mathfrak{G}_{\mathcal{H}}\left(B^{2}\right)$ be given by (3.1). Let $g_{1}$ and $g_{2}$ be nonzero elements in $\mathcal{O}(\mathcal{H})$ and for each $k \in\{1,2\}$, 
let $A_{g_{k}}$ be the $g_{k}^{\odot}$-admixable operator on $B$. Then

$$
\begin{aligned}
& \int_{B^{2}} \mathbf{F}\left(A_{g_{1}} w-A_{g_{2}} z, A_{g_{2}} w+A_{g_{1}} z\right) d(\nu \times \nu)(w, z) \\
= & \int_{B^{2}} \mathbf{F}\left(A_{s\left(g_{1}, g_{2}\right)} x, A_{s\left(g_{1}, g_{2}\right)} y\right) d(\nu \times \nu)(x, y) .
\end{aligned}
$$

It will be helpful to establish the following two lemmas before giving the proof of Theorem 3.1.

Lemma 3.2. Let $\mathcal{H}=\left\{h_{1}, \ldots, h_{n}\right\}$ be an orthogonal subset of $H$ and let $g_{1}$ and $g_{2}$ be nonzero elements in $\mathcal{O}(\mathcal{H})$. For each $j \in\{1, \ldots, n\}$, let $S_{j}, T_{j}: B \times B \rightarrow \mathbb{R}$ be given by

$$
S_{j}(w, z)=\left(h_{j}, A_{g_{1}} w\right)^{\sim}-\left(h_{j}, A_{g_{2}} z\right)^{\sim}
$$

and

$$
T_{j}(w, z)=\left(h_{j}, A_{g_{2}} w\right)^{\sim}+\left(h_{j}, A_{g_{1}} z\right)^{\sim},
$$

respectively, where $A_{g}$ denotes the $g^{\odot}$-admixable operator. Then

$$
\mathcal{R}=\left\{S_{1}, \ldots, S_{n}, T_{1}, \ldots, T_{n}\right\}
$$

is a set of independent Gaussian random variables.

Proof. We note that for each $k \in H,(k, x)^{\sim}$ has a Gaussian distribution with mean zero and variance $|k|^{2}$. Using this fact, we observe that for $k_{1}, k_{2} \in H$,

$$
\int_{B}\left(k_{1}, x\right)^{\sim}\left(k_{2}, x\right)^{\sim} d \nu(x)=\left\langle k_{1}, k_{2}\right\rangle .
$$

However, using (3.6) and Fubini's theorem, we have that for all $j, l \in\{1, \ldots, n\}$ with $j \neq l$,

$$
\begin{aligned}
& \int_{B^{2}} S_{j}(w, z) S_{l}(w, z) d(\nu \times \nu)(w, z) \\
= & \left\langle h_{j} \odot g_{1}, h_{l} \odot g_{1}\right\rangle+\left\langle h_{j} \odot g_{2}, h_{l} \odot g_{2}\right\rangle=0
\end{aligned}
$$

and

$$
\begin{aligned}
& \int_{B^{2}} T_{j}(w, z) T_{l}(w, z) d(\nu \times \nu)(w, z) \\
= & \left\langle h_{j} \odot g_{2}, h_{l} \odot g_{2}\right\rangle+\left\langle h_{j} \odot g_{1}, h_{l} \odot g_{1}\right\rangle=0 .
\end{aligned}
$$

Also, we have that for all $j, l \in\{1, \ldots, n\}$,

$$
\begin{aligned}
& \int_{B^{2}} S_{j}(w, z) T_{l}(w, z) d(\nu \times \nu)(w, z) \\
= & \left\langle h_{j} \odot g_{1}, h_{l} \odot g_{2}\right\rangle-\left\langle h_{j} \odot g_{2}, h_{l} \odot g_{1}\right\rangle=0,
\end{aligned}
$$

because

$$
\begin{aligned}
\left\langle h_{j} \odot g_{2}, h_{l} \odot g_{1}\right\rangle & =\left\langle h_{j} \odot g_{2} \odot g_{1}, h_{l}\right\rangle \\
& =\left\langle h_{j} \odot g_{1} \odot g_{2}, h_{l}\right\rangle=\left\langle h_{j} \odot g_{1}, h_{l} \odot g_{2}\right\rangle .
\end{aligned}
$$


From these facts above, we observe that for any $X, Y \in \mathcal{R}$ with $X \neq Y$, $\operatorname{Cov}(X, Y)=0$, which completes the proof of this lemma.

The following lemma follows from Lemma 3.2.

Lemma 3.3. Let $\mathcal{H}, g_{1}$ and $g_{2}$ be as in Lemma 3.2. Then the Gaussian random vectors

$$
R_{g_{1}, g_{2}}^{1}: B \times B \rightarrow \mathbb{R}^{n}, \quad R_{g_{1}, g_{2}}^{1}(w, z)=X_{\mathcal{H}}\left(A_{g_{1}} w\right)-X_{\mathcal{H}}\left(A_{g_{2}} z\right)
$$

and

$$
R_{g_{1}, g_{2}}^{2}: B \times B \rightarrow \mathbb{R}^{n}, \quad R_{g_{1}, g_{2}}^{2}(w, z)=X_{\mathcal{H}}\left(A_{g_{2}} w\right)+X_{\mathcal{H}}\left(A_{g_{1}} z\right)
$$

are independent. Furthermore, the covariance matrix of $R_{g_{1}, g_{2}}^{1}$ and $R_{g_{1}, g_{2}}^{2}$ is given by

$$
(\operatorname{Cov}(X, Y))_{X, Y \in \mathcal{R}}
$$

where $\mathcal{R}$ is given by (3.5) above.

We note that the determinant of the matrix $(\operatorname{Cov}(X, Y))_{X, Y \in \mathcal{R}}$ is given by

$$
\begin{aligned}
\operatorname{det}(\operatorname{Cov}(X, Y))_{X, Y \in \mathcal{R}} & =\operatorname{trace}(\operatorname{Cov}(X, Y))_{X, Y \in \mathcal{R}} \\
& =\sum_{j=1}^{n}\left(\left|h_{j} \odot g_{1}\right|^{2}+\left|h_{j} \odot g_{2}\right|^{2}\right) .
\end{aligned}
$$

We are now finally ready to give the proof of Theorem 3.1 .

Proof of Theorem 3.1. Using equations (3.3) and (3.4), we observe that for $(w, z) \in B \times B$,

$$
X_{\mathcal{H}}\left(A_{g_{1}} w\right)-X_{\mathcal{H}}\left(A_{g_{2}} z\right)=\left(S_{1}(w, z), \ldots, S_{n}(w, z)\right)
$$

and

$$
X_{\mathcal{H}}\left(A_{g_{2}} w\right)+X_{\mathcal{H}}\left(A_{g_{1}} z\right)=\left(T_{1}(w, z), \ldots, T_{n}(w, z)\right) .
$$

Thus, using these, (3.1), and Fubini's theorem, and applying Lemmas 3.3 and 2.4 , we obtain

$$
\begin{aligned}
& \int_{B \times B} \mathbf{F}\left(A_{g_{1}} w-A_{g_{2}} z, A_{g_{2}} w+A_{g_{1}} z\right) d(\nu \times \nu)(w, z) \\
= & \int_{B \times B} f\left(S_{1}(w, z), \ldots, S_{n}(w, z) ; T_{1}(w, z), \ldots, T_{n}(w, z)\right) d(\nu \times \nu)(w, z) \\
= & \left(\prod_{j=1}^{n} 2 \pi\left(\left|h_{j} \odot g_{1}\right|^{2}+\left|h_{j} \odot g_{2}\right|^{2}\right)\right)^{-1} \\
& \times \int_{\mathbb{R}^{2 n}} f(\vec{u} ; \vec{v}) \exp \left\{-\sum_{j=1}^{n} \frac{u_{j}^{2}+v_{j}^{2}}{2\left(\left|h_{j} \odot g_{1}\right|^{2}+\left|h_{j} \odot g_{2}\right|^{2}\right)}\right\} d \vec{u} d \vec{v} .
\end{aligned}
$$


Now, let $\beta_{j}=h_{j} \odot s\left(g_{1}, g_{2}\right)$ for each $j \in\{1, \ldots, n\}$. Then we have that for all $j, l \in\{1, \ldots, n\}$ with $j \neq l$,

$$
\begin{aligned}
\left\langle\beta_{j}, \beta_{l}\right\rangle & =\left\langle h_{j} \odot s\left(g_{1}, g_{2}\right), h_{l} \odot s\left(g_{1}, g_{2}\right)\right\rangle \\
& =\left\langle h_{j} \odot s\left(g_{1}, g_{2}\right) \odot s\left(g_{1}, g_{2}\right), h_{l}\right\rangle \\
& =\left\langle h_{j} \odot\left(g_{1}^{\odot 2}+g_{2}^{\odot 2}\right), h_{l}\right\rangle \\
& =\left\langle h_{j} \odot g_{1}^{\odot 2}, h_{l}\right\rangle+\left\langle h_{j} \odot g_{2}^{\odot 2}, h_{l}\right\rangle \\
& =\left\langle h_{j} \odot g_{1}, h_{l} \odot g_{1}\right\rangle+\left\langle h_{j} \odot g_{2}, h_{l} \odot g_{2}\right\rangle=0
\end{aligned}
$$

and that for each $j \in\{1, \ldots, n\}$,

$$
\begin{aligned}
\left|\beta_{j}\right|^{2} & =\left|h_{j} \odot s\left(g_{1}, g_{2}\right)\right|^{2}=\left\langle h_{j} \odot s\left(g_{1}, g_{2}\right), h_{j} \odot s\left(g_{1}, g_{2}\right)\right\rangle \\
& =\left\langle h_{j} \odot g_{1}, h_{j} \odot g_{1}\right\rangle+\left\langle h_{j} \odot g_{2}, h_{j} \odot g_{2}\right\rangle \\
& =\left|h_{j} \odot g_{1}\right|^{2}+\left|h_{j} \odot g_{2}\right|^{2} .
\end{aligned}
$$

Hence, from (3.8) and (3.9), we see that $\mathcal{B}=\left\{\beta_{1}, \ldots, \beta_{n}\right\}$ is an orthogonal set in $H$ and that the stochastic inner products

$$
\left(\beta_{j}, x\right)^{\sim}=\left(h_{j} \odot s\left(g_{1}, g_{2}\right), x\right)^{\sim}=\left(h_{j}, A_{s\left(g_{1}, g_{2}\right)} x\right)^{\sim}, j \in\{1, \ldots, n\}
$$

form a set of independent Gaussian random variables on $B$ with mean zero and variance $\left|h_{j} \odot g_{1}\right|^{2}+\left|h_{j} \odot g_{2}\right|^{2}$. Also, using Fubini's theorem and (2.7), we obtain

$$
\begin{aligned}
& \int_{B^{2}} \mathbf{F}\left(A_{s\left(g_{1}, g_{2}\right)} x, A_{s\left(g_{1}, g_{2}\right)} y\right) d(\nu \times \nu)(x, y) \\
= & \int_{B^{2}} f\left(\left(\beta_{1}, x\right)^{\sim}, \ldots,\left(\beta_{n}, x\right)^{\sim} ;\left(\beta_{1}, y\right)^{\sim}, \ldots,\left(\beta_{n}, y\right)^{\sim}\right) d(\nu \times \nu)(x, y) \\
= & \int_{B}\left[\int_{B} f\left(X_{\mathcal{B}}(x) ; X_{\mathcal{B}}(y)\right) d \nu(x)\right] d \nu(y) \\
= & \left(\prod_{j=1}^{n} 2 \pi\left(\left|h_{j} \odot g_{1}\right|^{2}+\left|h_{j} \odot g_{2}\right|^{2}\right)\right)^{-1 / 2} \\
& \times \int_{B}\left[\int_{\mathbb{R}^{n}} f\left(\vec{u} ; X_{\mathcal{B}}(y)\right) \exp \left\{-\sum_{j=1}^{n} \frac{u_{j}^{2}}{2\left(\left|h_{j} \odot g_{1}\right|^{2}+\left|h_{j} \odot g_{2}\right|^{2}\right)}\right\} d \vec{u}\right] d \nu(y) \\
= & \left(\prod_{j=1}^{n} 2 \pi\left(\left|h_{j} \odot g_{1}\right|^{2}+\left|h_{j} \odot g_{2}\right|^{2}\right)\right)^{-1} \\
& \times \int_{\mathbb{R}^{2 n}} f(\vec{u} ; \vec{v}) \exp \left\{-\sum_{j=1}^{n} \frac{u_{j}^{2}+v_{j}^{2}}{2\left(\left|h_{j} \odot g_{1}\right|^{2}+\left|h_{j} \odot g_{2}\right|^{2}\right)}\right\} d \vec{u} d \vec{v} .
\end{aligned}
$$

Equation (3.2) now follows from equations (3.7) and (3.10). 


\section{Classical Wiener space}

Let $B$ be the space $C_{0}[0, T]$ of all real-valued continuous functions $x(t)$ on the compact interval $[0, T]$ with $x(0)=0$, and equip $B=C_{0}[0, T]$ with the uniform norm $\|x\|=\sup _{t \in[0, T]}|x(t)|$. Let $H=C_{0}^{\prime}[0, T]$ be the real separable infinite dimensional Hilbert space consisting of the absolutely continuous functions $h(t)$ such that $h(0)=0$ and whose derivative $D h \equiv d h / d t$ is in $L_{2}[0, T]$. The inner product on $H$ is given by

$$
\left\langle h_{1}, h_{2}\right\rangle=\int_{0}^{T} D h_{1}(s) D h_{2}(s) d s
$$

Let $\nu=m_{\mathrm{w}}$ be the classical Wiener measure characterized by

$$
m_{\mathrm{w}}(\{x: x(t) \leq a\})=\frac{1}{\sqrt{2 \pi t}} \int_{-\infty}^{a} \exp \left\{-\frac{u^{2}}{2 t}\right\} d u .
$$

Then $\left(C_{0}[0, T], C_{0}^{\prime}[0, T], m_{\mathrm{w}}\right)$ is one of the most important examples of an abstract Wiener space. For more details, see [4, 13].

Let $I$ be the unitary operator from $L_{2}[0, T]$ onto $C_{0}^{\prime}[0, T]$ given by $I v(t)=$ $\int_{0}^{t} v(s) d s$ for $v \in L_{2}[0, T]$. Let $S[0, T]$ be the space of real-valued functions on $[0, T]$ which are continuous except for a finite number of finite jump discontinuities and are of bounded variation on $[0, T]$, and let

$$
C_{0}^{*}[0, T]=\{I v: v \in S[0, T]\} .
$$

For any $h \in C_{0}^{\prime}[0, T]$ and $g \in C_{0}^{*}[0, T]$, let the operation $\odot$ between $C_{0}^{\prime}[0, T]$ and $C_{0}^{*}[0, T]$ be defined by

$$
h \odot g=I(D h D g),
$$

where $D h D g$ denotes the pointwise multiplication of the functions $D h$ and $D g$. Then $\left(C_{0}^{*}[0, T], \odot\right)$ is a commutative algebra with the identity $e(t)=t$.

It is easy to verify that $\left\{x(t):(x, t) \in C_{0}[0, T] \times[0, T]\right\}$ is a standard Wiener process on the probability space $\left(C_{0}[0, T], \mathcal{B}\left(C_{0}[0, T]\right), m_{\mathrm{w}}\right)$.

Note that if $\left\{e_{n}\right\}_{n=1}^{\infty}$ is a complete orthonormal set of functions in $C_{0}^{\prime}[0, T]$, then $\left\{D e_{n}\right\}_{n=1}^{\infty}$ is also a complete orthonormal set of functions in $L_{2}[0, T]$ and $(h, x)^{\sim}$ equals the Paley-Wiener-Zygmund stochastic integral $\int_{0}^{T} D h(t) \tilde{d} x(t)$ for each $h \in C_{0}^{\prime}[0, T]$ and $m_{\mathrm{w}}$-a.e. $x \in C_{0}[0, T]$, see $[11,15,16]$.

Let $g \in C_{0}^{\prime}[0, T]$ with $|g|=\sqrt{\langle g, g\rangle}>0$. Then, the stochastic integral

$$
\mathcal{Z}_{g}(x, t)=\int_{0}^{t} D g(s) \tilde{d} x(s), \quad t \in[0, T]
$$

which was introduced by Park and Skoug in [17], is a Gaussian process with mean zero and covariance function

$$
\int_{C_{0}[0, T]} \mathcal{Z}_{g}(x, s) \mathcal{Z}_{g}(x, t) d m_{\mathrm{w}}(x)=\int_{0}^{\min \{s, t\}}(D g(u))^{2} d u
$$


In addition, $\mathcal{Z}_{g}(x, t)$ is stochastically continuous in $t$ on $[0, T]$. For more detailed information about this process, see $[5,8,18,19]$. Furthermore, if $g$ is an element of $C_{0}^{*}[0, T]$, then for all $x \in C_{0}[0, T], \mathcal{Z}_{g}(x, t)$ is continuous in $t$, and so $\mathcal{Z}_{g}(x, \cdot)$ is in $C_{0}[0, T]$.

From [5, Lemma 1], we note that for each $h \in C_{0}^{\prime}[0, T]$ and each $g \in C_{0}^{\prime}[0, T]$ with $D g \in L_{\infty}[0, T]$,

$$
\int_{0}^{T} D h(t) \tilde{d} \mathcal{Z}_{g}(x, t)=\int_{0}^{T} D h(t) D g(t) \tilde{d} x(t)
$$

for $m_{\mathrm{w}}$-a.e. $x \in C_{0}[0, T]$. We note that $D g$ is an element of $L_{\infty}[0, T]$ for every $g \in C_{0}^{*}[0, T]$.

Given $g \in C_{0}^{*}[0, T]$, define an operator $A_{g}^{\mathrm{w}}: C_{0}[0, T] \rightarrow C_{0}[0, T]$ by

$$
A_{g}^{\mathrm{w}} x=\mathcal{Z}_{g}(x, \cdot) .
$$

Then, for all $h \in C_{0}^{\prime}[0, T]$,

$$
\left(h, A_{g}^{\mathrm{w}} x\right)^{\sim}=\int_{0}^{T} D h(t) \tilde{d} \mathcal{Z}_{g}(x, t)=\int_{0}^{T} D h(t) D g(t) \tilde{d} x(t)=(h \odot g, x)^{\sim} .
$$

Thus, $A_{g}^{\mathrm{w}}$ is $g^{\odot}$-admixable in view of Definition 2.1 above.

We will now introduce the family of functions $\Gamma \equiv\left\{\gamma_{\tau}: \tau \in[0, T]\right\}$ from $C_{0}^{\prime}[0, T]$ :

$$
\gamma_{\tau}(s)= \begin{cases}s, & s \in[0, \tau] \\ \tau, & s \in[\tau, T]\end{cases}
$$

These functions have the reproducing property,

$$
\left\langle h, \gamma_{\tau}\right\rangle=\int_{0}^{T} D h(s) D \gamma_{\tau}(s) d s=\int_{0}^{T} D h(s) \chi_{[0, \tau]}(s) d s=\int_{0}^{\tau} D h(s) d s=h(\tau)
$$

for all $h \in C_{0}^{\prime}[0, T]$. In fact, $\Gamma \subset C_{0}^{*}[0, T]$.

From the observations above, we obtain the following proposition.

Proposition 4.1. For $\tau \in[0, T]$, let $\gamma_{\tau}$ be given by equation (4.3) and for a function $\theta(\cdot)$ of bounded variation on $[0, T]$, let

$$
\Theta_{c}(t)=\int_{0}^{t} \cos \theta(s) d s, \quad 0 \leq t \leq T
$$

and

$$
\Theta_{s}(t)=\int_{0}^{t} \sin \theta(s) d s, \quad 0 \leq t \leq T .
$$

Then $\Theta_{c}$ and $\Theta_{s}$ are elements of $C_{0}^{*}[0, T]$, and for each $\tau \in[0, T]$,

$$
\left(\gamma_{\tau}, A_{\Theta_{c}}^{\mathrm{w}} x\right)^{\sim}=\int_{0}^{\tau} \cos \theta(s) d x(s)=\mathcal{Z}_{\Theta_{c}}(x, \tau)
$$


and

$$
\left(\gamma_{\tau}, A_{\Theta_{s}}^{\mathrm{w}} x\right)^{\sim}=\int_{0}^{\tau} \sin \theta(s) d x(s)=\mathcal{Z}_{\Theta_{s}}(x, \tau),
$$

where $A_{\Theta_{c}}^{\mathrm{w}}$ and $A_{\Theta_{s}}^{\mathrm{w}}$ are admixable operators given by equation (4.2) on Wiener space $C_{0}[0, T]$. Furthermore,

$$
\left(\Theta_{c}, A_{\gamma_{\tau}}^{\mathrm{w}} x\right)^{\sim}=\left(\gamma_{\tau}, A_{\Theta_{c}}^{\mathrm{w}} x\right)^{\sim}
$$

and

$$
\left(\Theta_{s}, A_{\gamma_{\tau}}^{\mathrm{w}} x\right)^{\sim}=\left(\gamma_{\tau}, A_{\Theta_{s}}^{\mathrm{w}} x\right)^{\sim}
$$

are elements of $C_{0}[0, T]$ as a function of $\tau$.

Remark 4.2. Let $\mathcal{M}$ be the $\sigma$-algebra of all $m_{\mathrm{w}}$-measurable subsets of $C_{0}[0, T]$. Then $\left(C_{0}[0, T], \mathcal{M}, m_{\mathrm{w}}\right)$ is a complete measure space. It is well known that $\mathcal{M}$ coincides with $\sigma\left(\mathcal{B}\left(C_{0}[0, T]\right)\right)$, the completion of $\mathcal{B}\left(C_{0}[0, T]\right)$. Thus, for any $\mathcal{M}$-measurable functional $F$, there exists a $\mathcal{B}\left(C_{0}[0, T]\right)$-measurable functional $F^{*}$ such that $F(x)=F^{*}(x)$ for $m_{\mathrm{w}}$-a.e. $x$ in $\left(C_{0}[0, T], \mathcal{B}\left(C_{0}[0, T]\right)\right)$ (see $[6$, Proposition 2.2.5]).

Proof of Theorem 1.1. Let $n$ be any positive integer, and let $0=\tau_{0}<\tau_{1}<$ $\cdots<\tau_{n} \leq T$ be any partition of $[0, T]$. In view of Remark 4.2 and the proofs which are given in Sections 2-6 in [1], it will suffice to show that equation (1.2) holds for any tame function $\mathbf{F}$ of the form

$$
\begin{aligned}
\mathbf{F}(x, y)=f\left(x\left(\tau_{1}\right), x\left(\tau_{2}\right)-\right. & x\left(\tau_{1}\right), \ldots, x\left(\tau_{n}\right)-x\left(\tau_{n-1}\right) \\
& \left.y\left(\tau_{1}\right), y\left(\tau_{2}\right)-y\left(\tau_{1}\right), \ldots, y\left(\tau_{n}\right)-y\left(\tau_{n-1}\right)\right),
\end{aligned}
$$

where $f: \mathbb{R}^{2 n} \rightarrow \mathbb{C}$ is a Borel measurable function and is of class $L_{1}\left(\mathbb{R}^{2 n}\right)$.

Let $\odot$ be defined by (4.1) between $C_{0}^{\prime}[0, T]$ and $C_{0}^{*}[0, T]$. Let $\Theta_{c}$ and $\Theta_{s}$ be given by (4.4) and (4.5), respectively, and let $A_{\Theta_{c}}^{\mathrm{w}}$ and $A_{\Theta_{s}}^{\mathrm{w}}$ be admixable operators corresponding to $\Theta_{c}$ and $\Theta_{s}$. Also for each $j \in\{1, \ldots, n\}$, let

$$
h_{j}(t)=\int_{0}^{t} \chi_{\left[\tau_{j-1}, \tau_{j}\right]}(s) d s
$$

on $[0, T]$. Then $\mathcal{H}=\left\{h_{1}, \ldots, h_{n}\right\}$ is an orthogonal set in $C_{0}^{\prime}[0, T]$ and the functional $\mathbf{F}$ given by (4.6) can be rewritten as

$$
\begin{aligned}
\mathbf{F}(x, y) & =f\left(\left(h_{1}, x\right)^{\sim}, \ldots,\left(h_{n}, x\right)^{\sim} ;\left(h_{1}, y\right)^{\sim}, \ldots,\left(h_{n}, y\right)^{\sim}\right) \\
& \equiv f\left((\vec{h}, x)^{\sim} ;(\vec{h}, y)^{\sim}\right) .
\end{aligned}
$$

Clearly, $\mathcal{H} \odot \Theta_{c}=\left\{h_{1} \odot \Theta_{c}, \ldots, h_{n} \odot \Theta_{c}\right\}$ and $\mathcal{H} \odot \Theta_{s}=\left\{h_{1} \odot \Theta_{s}, \ldots, h_{n} \odot \Theta_{s}\right\}$ are orthogonal sets in $C_{0}^{\prime}[0, T]$. Moreover, for each $j \in\{1, \ldots, n\}$,

$$
\begin{aligned}
\left(h_{j}, A_{\Theta_{c}}^{\mathrm{w}} w\right)^{\sim} & =\left(h_{j} \odot \Theta_{c}, w\right)^{\sim}=\int_{\tau_{j-1}}^{\tau_{j}} \cos \theta(s) d w(s) \\
& =\int_{0}^{\tau_{j}} \cos \theta(s) d w(s)-\int_{0}^{\tau_{j-1}} \cos \theta(s) d w(s)
\end{aligned}
$$


for $m_{\mathrm{w}}$-a.e. $w \in C_{0}[0, T]$, and

$$
\begin{aligned}
\left(h_{j}, A_{\Theta_{s}}^{\mathrm{w}} z\right)^{\sim} & =\left(h_{j} \odot \Theta_{s}, z\right)^{\sim}=\int_{\tau_{j-1}}^{\tau_{j}} \sin \theta(s) d z(s) \\
& =\int_{0}^{\tau_{j}} \sin \theta(s) d z(s)-\int_{0}^{\tau_{j-1}} \sin \theta(s) d z(s)
\end{aligned}
$$

for $m_{\mathrm{w}}$-a.e. $z \in C_{0}[0, T]$. Thus, using equation (1.1) together with (4.8) and (4.9), and the third expression of equation (4.7), the left side of (1.2) with $\mathbf{F}$ given by (4.6) is rewritten as

$$
\begin{aligned}
& \int_{C_{0}^{2}[0, T]} \mathbf{F}\left(T_{\theta}(w, z)\right) d\left(m_{\mathrm{w}} \times m_{\mathrm{w}}\right)(w, z) \\
= & \int_{C_{0}^{2}[0, T]} f\left(\left(\vec{h}, A_{\Theta_{c}}^{\mathrm{w}} w\right)^{\sim}-\left(\vec{h}, A_{\Theta_{s}}^{\mathrm{w}} z\right)^{\sim} ;\right. \\
= & \int_{C_{0}^{2}[0, T]} f\left(\left(\vec{h}, A_{\Theta_{s}}^{\mathrm{w}} w\right)^{\sim}+\left(\vec{h}, A_{\Theta_{c}}^{\mathrm{w}} z\right)^{\sim}\right) d\left(m_{\mathrm{w}} \times m_{\mathrm{w}}\right)(w, z) \\
= & \left.\left.\int_{C_{0}^{2}[0, T]}^{\mathrm{w}} w-A_{\Theta_{s}}^{\mathrm{w}} z\right)^{\sim} ;\left(\vec{h}, A_{\Theta_{s}}^{\mathrm{w}} w+A_{\Theta_{c}}^{\mathrm{w}} z\right)^{\sim}\right) d\left(m_{\mathrm{w}} \times m_{\mathrm{w}}\right)(w, z) \\
= & \left.\int_{C_{0}^{2}[0, T]} \mathbf{F}\left(A_{\Theta_{c}}^{\mathrm{w}} w-A_{\Theta_{c}}^{\mathrm{w}} w-A_{\Theta_{s}}^{\mathrm{w}} z\right) ; X_{\mathcal{H}}\left(A_{\Theta_{s}}^{\mathrm{w}} w+A_{\Theta_{c}}^{\mathrm{w}} z\right)\right) d\left(m_{\mathrm{w}} \times m_{\mathrm{w}}\right)(w, z)
\end{aligned}
$$

where $X_{\mathcal{H}}$ is given by equation (2.4) with $\mathcal{V}$ replaced with $\mathcal{H}$. Thus, using (3.2) with $A_{g_{1}}$ and $A_{g_{2}}$ replaced with $A_{\Theta_{c}}^{\mathrm{w}}$ and $A_{\Theta_{s}}^{\mathrm{w}}$ respectively, and equation (4.2), we obtain

$$
\begin{aligned}
& \int_{C_{0}^{2}[0, T]} \mathbf{F}\left(T_{\theta}(w, z)\right) d\left(m_{\mathrm{w}} \times m_{\mathrm{w}}\right)(w, z) \\
= & \int_{C_{0}^{2}[0, T]} \mathbf{F}\left(A_{s\left(\Theta_{c}, \Theta_{s}\right)}^{\mathrm{w}} x, A_{s\left(\Theta_{c}, \Theta_{s}\right)}^{\mathrm{w}} y\right) d\left(m_{\mathrm{w}} \times m_{\mathrm{w}}\right)(x, y) \\
= & \int_{C_{0}^{2}[0, T]} \mathbf{F}\left(\mathcal{Z}_{s\left(\Theta_{c}, \Theta_{s}\right)}(x, \cdot), \mathcal{Z}_{s\left(\Theta_{c}, \Theta_{s}\right)}(y, \cdot)\right) d\left(m_{\mathrm{w}} \times m_{\mathrm{w}}\right)(x, y) .
\end{aligned}
$$

We note that by equations (2.3), (2.2) and (4.1),

$$
\begin{aligned}
s^{\odot 2}\left(\Theta_{c}, \Theta_{s}\right)(t) & \equiv \Theta_{c}^{\odot 2}(t)+\Theta_{s}^{\odot 2}(t) \\
& =\int_{0}^{t} \cos ^{2} \theta(s) d s+\int_{0}^{t} \sin ^{2} \theta(s) d s=\int_{0}^{t} d s .
\end{aligned}
$$

But in view of condition (a5) of Definition 2.1 and (4.1), we see that either $s\left(\Theta_{c}, \Theta_{s}\right)(t)=t$ or $s\left(\Theta_{c}, \Theta_{s}\right)(t)=-t$, i.e., either $\mathcal{Z}_{s\left(\Theta_{c}, \Theta_{s}\right)}(x, \cdot)=x(\cdot)$ or 
$\mathcal{Z}_{s\left(\Theta_{c}, \Theta_{s}\right)}(x, \cdot)=-x(\cdot)$. But we know that for all $\mathcal{B}\left(C_{0}[0, T]\right)$-measurable functional $F$,

$$
\int_{C_{0}[0, T]} F(x) d m_{\mathrm{w}}(x)=\int_{C_{0}[0, T]} F(-x) d m_{\mathrm{w}}(x) .
$$

Hence, the last expression in equation (4.10) above can be rewritten as

$$
\int_{C_{0}^{2}[0, T]} \mathbf{F}(x, y) d\left(m_{\mathrm{w}} \times m_{\mathrm{w}}\right)(x, y) .
$$

Thus we obtain the desired result.

\section{5. $g^{\odot}$-admix-Wiener transform}

In this section, we define a transform on abstract Wiener space $B$ and investigate its algebraic properties. Let $(B, H, \nu)$ be an abstract Wiener space, and let $F: B \rightarrow \mathbb{C}$ be a Wiener measurable functional. For $g \in B^{*}$, we define the $g^{\odot}$-admix-Wiener transform, $T_{g}^{\mathrm{w}}(F)$ of $F$, by the formula

$$
T_{g}^{\mathrm{w}}(F)(y)=\int_{B} F\left(y+A_{g} x\right) d \nu(x),
$$

if it exists, where $A_{g}$ is the $g^{\odot}$-admixable operator.

We note that if $T_{g}^{\mathrm{w}}\left(F_{1}\right)$ exists and if $F_{1}=F_{2}$ for $\nu$-a.e., then $T_{g}^{\mathrm{w}}\left(F_{2}\right)$ exists and $T_{g}^{\mathrm{w}}\left(F_{2}\right)=T_{g}^{\mathrm{w}}\left(F_{1}\right)$ for $\nu$-a.e..

Given an orthogonal set $\mathcal{H}$ in $H$, let $\mathfrak{K}_{\mathcal{H}}^{(2)}(B)$ be the class of all functionals $F: B \rightarrow \mathbb{C}$ given by

$$
F(x)=f\left(\left(h_{1}, x\right)^{\sim}, \ldots,\left(h_{n}, x\right)^{\sim}\right) \equiv f\left(X_{\mathcal{H}}(x)\right)
$$

for $\nu$-a.e. $x \in B$, where $f: \mathbb{R}^{n} \rightarrow \mathbb{C}$ is Borel measurable and is of class $L_{2}\left(\mathbb{R}^{n}\right)$.

Throughout this section, for convenience, we use the following notations: for a finite sequence $\mathcal{G}_{m}=\left(g_{1}, \ldots, g_{m}\right)$ in $H$, let

$$
s\left(\mathcal{G}_{m}\right) \equiv s\left(g_{1}, \ldots, g_{m}\right)=\sqrt[\odot]{\sum_{j=1}^{m} g_{j}^{\odot 2}} .
$$

Also, for $\mathcal{H}=\left\{h_{1}, \ldots, h_{n}\right\} \subset H$ and $g \in B^{*}-\{0\}$, let

$$
\begin{aligned}
\psi(\mathcal{H} \odot g ; \vec{r}) & \equiv \psi\left(\mathcal{H} \odot g ; r_{1}, \ldots, r_{n}\right) \\
& =\left(\prod_{j=1}^{n} 2 \pi\left|h_{j} \odot g\right|^{2}\right)^{-1 / 2} \int_{\mathbb{R}^{n}} f(\vec{u}) \exp \left\{-\sum_{j=1}^{n} \frac{\left(u_{j}-r_{j}\right)^{2}}{\left|h_{j} \odot g\right|^{2}}\right\} d \vec{u} .
\end{aligned}
$$

For all $g \in \mathcal{O}(\mathcal{H}), \psi(\mathcal{H} \odot g ; \vec{r})$ is in $L_{2}\left(\mathbb{R}^{n}\right)$, as a function of $\vec{r}$, by [9, Lemma 1.1]. From this fact, we obtain the following proposition. 
Proposition 5.1. Let $F \in \mathfrak{K}_{\mathcal{H}}^{(2)}(B)$ be given by equation (5.1). Then for all nonzero $g \in \mathcal{O}(\mathcal{H})$, the $g^{\odot}$-admix-Wiener transform, $T_{g}^{\mathrm{w}}(F)$ exists, belongs to $\mathfrak{K}_{\mathcal{H}}^{(2)}(B)$ and is given by the formula

$$
T_{g}^{\mathrm{w}}(F)(y)=\psi\left(\mathcal{H} \odot g ; X_{\mathcal{H}}(y)\right)
$$

for $\nu$-a.e. $y \in B$, where $\psi$ is given by (5.3).

We now present a theorem that will play a key role in this section.

Theorem 5.2. Let $F$ be a complex-valued $\nu$-integrable functional on $B$ given by equation (5.1), and let $g_{1}$ and $g_{2}$ be nonzero elements in $\mathcal{O}(\mathcal{H})$. Then

$$
\int_{B^{2}} F\left(A_{g_{1}} w+A_{g_{2}} z\right) d(\nu \times \nu)(w, z)=\int_{B} F\left(A_{s\left(g_{1}, g_{2}\right)} x\right) d \nu(x)
$$

where $A_{g}$ denotes the $g^{\odot}$-admixable operator.

Proof. Let $P: B^{2} \rightarrow B$ be the projection map given by $P(w, z)=z$. Then equation (5.4) follows from equation (3.2) with $\mathbf{F}$ replaced with $\mathbf{F} \circ P$.

The following theorem follows immediately from Proposition 5.1 and Theorem 5.2.

Proposition 5.3. Let $F \in \mathfrak{K}_{\mathcal{H}}^{(2)}(B)$ be given by equation (5.1). Then for any nonzero elements $g_{1}$ and $g_{2}$ in $\mathcal{O}(\mathcal{H})$,

$$
\left(T_{g_{2}}^{\mathrm{w}}\left(T_{g_{1}}^{\mathrm{w}}(F)\right)(y)=T_{s\left(g_{1}, g_{2}\right)}^{\mathrm{w}}(F)(y)\right.
$$

for $\nu$-a.e. $y \in B$.

Let $T_{0}^{\mathrm{w}}$ denote the identity transform; i.e., $T_{0}^{\mathrm{w}}(F)=F$, and let

$$
\mathfrak{T}\left[\mathfrak{K}_{\mathcal{H}}^{(2)}(B) ; \mathcal{O}(\mathcal{H})\right]=\left\{T_{g}^{\mathrm{w}}: g \in \mathcal{O}(\mathcal{H})\right\}
$$

be the class of admix-Wiener transforms acting on $\mathfrak{K}_{\mathcal{H}}^{(2)}(B)$.

By Propositions 5.1 and 5.3, we see that for all $g_{1}, g_{2} \in \mathcal{O}(\mathcal{H})$ and all $F \in$ $\mathfrak{K}_{\mathcal{H}}^{(2)}(B)$,

$$
\left(T_{g_{2}}^{\mathrm{w}} \circ T_{g_{1}}^{\mathrm{w}}\right)(F) \equiv\left(T_{g_{2}}^{\mathrm{w}}\left(T_{g_{1}}^{\mathrm{w}}(F)\right)=T_{s\left(g_{1}, g_{2}\right)}^{\mathrm{w}}(F)\right.
$$

is in $\mathfrak{K}_{\mathcal{H}}^{(2)}(B)$. Because for all $g_{1}, g_{2}, g_{3} \in \mathcal{O}(\mathcal{H})$,

$$
s\left(s\left(g_{3}, g_{2}\right), g_{1}\right)=s\left(g_{3}, g_{2}, g_{1}\right)=s\left(g_{3}, s\left(g_{2}, g_{1}\right)\right),
$$

we see that the composition o of admix-Wiener transforms is associative. Also, because $s\left(g_{1}, g_{2}\right)=s\left(g_{2}, g_{1}\right)$, we see that $\left(T_{g_{2}}^{\mathrm{w}} \circ T_{g_{1}}^{\mathrm{w}}\right)(F)=\left(T_{g_{1}}^{\mathrm{w}} \circ T_{g_{2}}^{\mathrm{w}}\right)(F)$, and clearly $\left(T_{0}^{\mathrm{w}} \circ T_{g_{1}}^{\mathrm{w}}\right)(F)=T_{g_{1}}^{\mathrm{w}}(F)$ for any $g_{1}, g_{2} \in \mathcal{O}(\mathcal{H})$ and every $F \in \mathfrak{K}^{(2)}(B)$. Thus, we have the following proposition.

Proposition 5.4. The space $\left(\mathfrak{T}\left[\mathfrak{K}_{\mathcal{H}}^{(2)}(B) ; \mathcal{O}(\mathcal{H})\right], \circ\right)$ is a commutative monoid. Furthermore, the monoid $\mathfrak{T}\left[\mathfrak{K}_{\mathcal{H}}^{(2)}(B) ; \mathcal{O}(\mathcal{H})\right]$ acts on the space $\mathfrak{K}_{\mathcal{H}}^{(2)}(B)$ in the sense that $\left(T_{g}^{\mathrm{w}}, F\right) \mapsto T_{g}^{\mathrm{w}}(F)$. 
Next, let $S_{\mathrm{f}}(\mathcal{O}(\mathcal{H}))$ be the set of all finite sequences in $\mathcal{O}(\mathcal{H})$, and let

$$
\mathfrak{T}\left[\mathfrak{K}_{\mathcal{H}}^{(2)}(B) ; S_{\mathrm{f}}(\mathcal{O}(\mathcal{H}))\right]=\left\{T_{s\left(\mathcal{G}_{m}\right)}^{\mathrm{w}}: \mathcal{G}_{m} \in S_{\mathrm{f}}(\mathcal{O}(\mathcal{H}))\right\}
$$

Using the fact that for any $\mathcal{G}_{m} \in S_{\mathrm{f}}(\mathcal{O}(\mathcal{H})), s\left(\mathcal{G}_{m}\right) \in \mathcal{O}(\mathcal{H}) \subset S_{\mathrm{f}}(\mathcal{O}(\mathcal{H}))$, we know that the classes $\mathfrak{T}\left[\mathfrak{K}_{\mathcal{H}}^{(2)}(B) ; \mathcal{O}(\mathcal{H})\right]$ and $\mathfrak{T}\left[\mathfrak{K}_{\mathcal{H}}^{(2)}(B) ; S_{\mathrm{f}}(\mathcal{O}(\mathcal{H}))\right]$ coincide as sets. However, we will consider another operation, $\circledast$, on $\mathfrak{T}\left[\mathfrak{K}_{\mathcal{H}}^{(2)}(B) ; S_{\mathrm{f}}(\mathcal{O}(\mathcal{H}))\right]$, defined as follows: for any $\mathcal{G}_{m_{1}}=\left(g_{1}^{1}, \ldots, g_{m_{1}}^{1}\right)$ and $\mathcal{G}_{m_{2}}=\left(g_{1}^{2}, \ldots, g_{m_{2}}^{2}\right)$ in $S_{\mathrm{f}}(\mathcal{O}(\mathcal{H}))$, let

$$
\begin{aligned}
\mathcal{G}_{m_{1}} \wedge \mathcal{G}_{m_{2}} & \equiv\left(g_{1}^{1}, \ldots, g_{m_{1}}^{1}\right) \wedge\left(g_{1}^{2}, \ldots, g_{m_{2}}^{2}\right) \\
& =\left(g_{1}^{1}, \ldots, g_{m_{1}}^{1}, g_{1}^{2}, \ldots, g_{m_{2}}^{2}\right),
\end{aligned}
$$

and for $T_{s\left(\mathcal{G}_{m_{1}}\right)}^{\mathrm{w}}$ and $T_{s\left(\mathcal{G}_{m_{2}}\right)}^{\mathrm{w}}$ in $\mathfrak{T}\left[\mathfrak{K}_{\mathcal{H}}^{(2)}(B) ; S_{\mathrm{f}}(\mathcal{O}(\mathcal{H}))\right]$, let

$$
T_{s\left(\mathcal{G}_{m_{1}}\right)}^{\mathrm{w}} \circledast T_{s\left(\mathcal{G}_{m_{2}}\right)}^{\mathrm{w}}=T_{s\left(\mathcal{G}_{m_{1}} \wedge \mathcal{G}_{m_{2}}\right)}^{\mathrm{w}} .
$$

Because

$$
\begin{aligned}
& s\left(s\left(\mathcal{G}_{m_{1}}\right), s\left(\mathcal{G}_{m_{2}}\right)\right)=\sqrt[\odot]{s\left(\mathcal{G}_{m_{1}}\right) \odot 2+s\left(\mathcal{G}_{m_{2}}\right) \odot 2} \\
& =\sqrt[\odot]{\sum_{j=1}^{m_{1}} g_{j}^{1 \odot 2}+\sum_{j=1}^{m_{2}} g_{j}^{2 \odot 2}} \\
& =s\left(\mathcal{G}_{m_{1}} \wedge \mathcal{G}_{m_{2}}\right) \text {, }
\end{aligned}
$$

the operation $\circledast$ given by $(5.7)$ is well defined in view of equation (5.5).

Proposition 5.5. The space $\left(\mathfrak{T}\left[\mathfrak{K}_{\mathcal{H}}^{(2)}(B) ; S_{\mathrm{f}}(\mathcal{O}(\mathcal{H}))\right], \circledast\right)$ is a commutative monoid. Furthermore, the monoid $\mathfrak{T}\left[\mathfrak{K}_{\mathcal{H}}^{(2)}(B) ; S_{\mathrm{f}}(\mathcal{O}(\mathcal{H}))\right]$ acts on the space $\mathfrak{K}_{\mathcal{H}}^{(2)}(B)$ in the sense that $\left(T_{s(\mathcal{G})}^{\mathrm{w}}, F\right) \mapsto T_{s(\mathcal{G})}^{\mathrm{w}}(F)$.

Remark 5.6. The operation $\circledast$ is a semigroup action of $\mathfrak{T}\left[\mathfrak{K}_{\mathcal{H}}^{(2)}(B) ; S_{\mathrm{f}}(\mathcal{O}(\mathcal{H}))\right]$, i.e., $\mathfrak{T}\left[\mathfrak{K}_{\mathcal{H}}^{(2)}(B) ; S_{\mathrm{f}}(\mathcal{O}(\mathcal{H}))\right]$ is a transform semigroup.

In fact, the sequence space $S_{\mathrm{f}}(\mathcal{O}(\mathcal{H}))$ is a semigroup under the operation $\wedge$, given by (5.6) above. Thus we see clearly that for any $\left(g_{1}, \ldots, g_{m}\right) \in S_{\mathrm{f}}(\mathcal{O}(\mathcal{H}))$ and any permutation $\pi$ of $\{1, \ldots, m\}$,

$$
s\left(g_{1}, \ldots, g_{m}\right)=s\left(g_{\pi(1)}, \ldots, g_{\pi(m)}\right) .
$$

Define an equivalence relation $\stackrel{\mathrm{s}}{\sim}$ on $S_{\mathrm{f}}(\mathcal{O}(\mathcal{H}))$ as follows: for $\mathcal{G}_{m_{1}}$ and $\mathcal{G}_{m_{2}}$ in $S_{\mathrm{f}}(\mathcal{O}(\mathcal{H}))$,

Also, let

$$
\mathcal{G}_{m_{1}} \stackrel{\mathrm{s}}{\sim} \mathcal{G}_{m_{2}} \Longleftrightarrow s\left(\mathcal{G}_{m_{1}}\right)=s\left(\mathcal{G}_{m_{2}}\right)
$$

$$
\widetilde{S_{\mathrm{f}}} \equiv S_{\mathrm{f}}(\mathcal{O}(\mathcal{H})) / \stackrel{\mathrm{s}}{\sim}=\left\{\left[\mathcal{G}_{m}\right]_{\mathrm{s}}: \mathcal{G}_{m} \in S_{\mathrm{f}}(\mathcal{O}(\mathcal{H}))\right\}
$$

be the quotient set of $S_{\mathrm{f}}(\mathcal{O}(\mathcal{H}))$ by $\stackrel{\mathrm{s}}{\sim}$. Then from equations (5.2) and (5.8), we see that $\widetilde{S_{\mathrm{f}}}$ is the quotient semigroup under the operation $\wedge$, defined by

$$
\left[\mathcal{G}_{m_{1}}\right]_{\mathrm{s}} \wedge\left[\mathcal{G}_{m_{2}}\right]_{\mathrm{s}}=\left[\mathcal{G}_{m_{1}} \wedge \mathcal{G}_{m_{2}}\right]_{\mathrm{s}}
$$


Define a relation on $\mathfrak{T}\left[\mathfrak{K}_{\mathcal{H}}^{(2)}(B) ; S_{\mathrm{f}}(\mathcal{O}(\mathcal{H}))\right]$ as follows: for $T_{s\left(\mathcal{G}_{m_{1}}\right)}^{\mathrm{w}}$ and $T_{s\left(\mathcal{G}_{m_{2}}\right)}^{\mathrm{w}}$ in $\mathfrak{T}\left[\mathfrak{K}_{\mathcal{H}}^{(2)}(B) ; S_{\mathrm{f}}(\mathcal{O}(\mathcal{H}))\right]$,

$$
T_{s\left(\mathcal{G}_{m_{1}}\right)}^{\mathrm{w}} \stackrel{\mathrm{t}}{\sim} T_{s\left(\mathcal{G}_{m_{2}}\right)}^{\mathrm{w}} \Longleftrightarrow \mathcal{G}_{m_{1}} \stackrel{\mathrm{s}}{\sim} \mathcal{G}_{m_{2}} .
$$

From equations (5.5), (5.2), and (5.8), we see that for every $\left(g_{1}, \ldots, g_{m}\right) \in$ $S_{\mathrm{f}}(\mathcal{O}(\mathcal{H}))$ and any permutation $\pi$ of $\{1, \ldots, m\}$,

$$
T_{s\left(g_{1}, \ldots, g_{m}\right)}^{\mathrm{w}}(F)=T_{s\left(g_{\pi(1)}, \ldots, g_{\pi(m)}\right)}^{\mathrm{w}}(F) .
$$

Thus, the relation $\stackrel{\mathrm{t}}{\sim}$ is a well-defined equivalence relation, and so we can obtain the quotient semigroup

$$
\begin{aligned}
\widetilde{\mathfrak{T}} & \equiv \mathfrak{T}\left[\mathfrak{K}_{\mathcal{H}}^{(2)}(B) ; S_{\mathrm{f}}(\mathcal{O}(\mathcal{H}))\right] / \stackrel{\mathrm{t}}{\sim} \\
& =\left\{\left[T_{s\left(\mathcal{G}_{m}\right)}^{\mathrm{w}}\right]_{\mathrm{t}}: T_{s\left(\mathcal{G}_{m}\right)}^{\mathrm{w}} \in \mathfrak{T}\left[\mathfrak{K}_{\mathcal{H}}^{(2)}(B) ; S_{\mathrm{f}}(\mathcal{O}(\mathcal{H}))\right]\right\}
\end{aligned}
$$

under the operation $\circledast$, given by

$$
\left[T_{s\left(\mathcal{G}_{m_{1}}\right)}^{\mathrm{w}}\right]_{\mathrm{t}} \circledast\left[T_{s\left(\mathcal{G}_{m_{2}}\right)}^{\mathrm{w}}\right]_{\mathrm{t}}=\left[T_{s\left(\mathcal{G}_{m_{1}} \wedge \mathcal{G}_{m_{2}}\right)}^{\mathrm{w}}\right]_{\mathrm{t}} .
$$

Theorem 5.7. Define a map $\Xi:(\widetilde{\mathfrak{T}}, \circledast) \rightarrow\left(\widetilde{S_{\mathrm{f}}}, \wedge\right)$ by

$$
\Xi\left(\left[T_{s\left(\mathcal{G}_{m}\right)}^{\mathrm{w}}\right]_{\mathrm{t}}\right)=\left[\mathcal{G}_{m}\right]_{\mathrm{s}} .
$$

Then $\Xi$ is a semigroup isomorphism.

Proof. It follows from (5.9) and (5.10) that

$$
\begin{aligned}
\Xi\left(\left[T_{s\left(\mathcal{G}_{m_{1}}\right)}^{\mathrm{w}}\right]_{\mathrm{t}} \circledast\left[T_{s\left(\mathcal{G}_{m_{2}}\right)}^{\mathrm{w}}\right]_{\mathrm{t}}\right) & =\Xi\left(\left[T_{s\left(\mathcal{G}_{m_{1}} \wedge \mathcal{G}_{m_{2}}\right)}^{\mathrm{w}}\right]_{\mathrm{t}}\right) \\
& =\left[\mathcal{G}_{m_{1}} \wedge \mathcal{G}_{m_{2}}\right]_{\mathrm{s}} \\
& =\left[\mathcal{G}_{m_{1}}\right]_{\mathrm{s}} \wedge\left[\mathcal{G}_{m_{2}}\right]_{\mathrm{s}} \\
& =\Xi\left(\left[T_{s\left(\mathcal{G}_{m_{1}}\right)}^{\mathrm{w}}\right]_{\mathrm{t}}\right) \circledast \Xi\left(\left[T_{s\left(\mathcal{G}_{m_{2}}\right)}^{\mathrm{w}}\right]_{\mathrm{t}}\right) .
\end{aligned}
$$

Clearly, the map given by equation (5.11) is bijective.

\section{References}

[1] J. E. Bearman, Rotations in the product of two Wiener spaces, Proc. Amer. Math. Soc. 3 (1952), no. 1, 129-137.

[2] R. H. Cameron and D. A. Storvick, An operator valued Yeh-Wiener integral, and a Wiener integral equation, Indiana Univ. Math. J. 25 (1976), no. 3, 235-258.

[3] J. G. Choi and S. J. Chang, A rotation of admixable operators on abstract Wiener space with applications, J. Funct. Space Appl. 2013 (2013), Article ID 671909, 12 pages.

[4] D. M. Chung, Scale-invariant measurability in abstract Wiener spaces, Pacific J. Math. 130 (1987), no. 1, 27-40.

[5] D. M. Chung, C. Park, and D. Skoug, Generalized Feynman integrals via conditional Feynman integrals, Michigan Math. J. 40 (1993), no. 2, 377-391.

[6] D. L. Cohn, Measure Theory, Second edition, Birkhäuser Advanced Texts Basler Lehrbücher, Birkhäuser, Boston, 2013.

[7] L. Gross, Abstract Wiener spaces, Proceedings of the 5th Berkeley Symposium on Mathematical Statistics and Probability, vol. 2, pp. 31-42, University of California Press, Berkeley, 1965 
[8] T. Huffman, C. Park, and D. Skoug, Generalized transforms and convolutions, Int. J. Math. Math. Sci. 20 (1997), no. 1, 19-32.

[9] G. W. Johnson and D. L. Skoug, The Cameron-Storvick function space integral: An $L\left(L_{p}, L_{p^{\prime}}\right)$ theory, Nagoya Math. J. 60 (1976), 93-137.

[10] _ Scale-invariant measurability in Wiener space, Pacific J. Math. 83 (1979), no. 1, 157-176.

[11] Notes on the Feynman integral. II, J. Funct. Anal. 41 (1981), no. 3, 277-289.

[12] G. Kallianpur and C. Bromley, Generalized Feynman integrals using analytic continuation in several complex variables, Stochastic Analysis and Applications, pp. 217-267, Dekker, New York, 1984.

[13] H.-H. Kuo, Gaussian Measures in Banach Spaces, Lecture Notes in Math. 463, Springer, Berlin, 1975.

[14] Y.-J. Lee, Integral transforms of analytic functions on abstract Wiener spaces, J. Funct. Anal. 47 (1982), no. 2, 153-164.

[15] R. E. A. C. Paley, N. Wiener, and A. Zygmund, Notes on random functions, Math. Z. 37 (1933), no. 1, 647-668.

[16] C. Park and D. Skoug, A note on Paley-Wiener-Zygmund stochastic integrals, Proc. Amer. Math. Soc. 103 (1988), no. 2, 591-601.

[17] _ A Kac-Feynman integral equation for conditional Wiener integrals, J. Integral Equations Appl. 3 (1991), no. 3, 411-427.

[18] _ Generalized Feynman integrals: The $\mathcal{L}\left(L_{2}, L_{2}\right)$ theory, Rocky Mountain J. Math. 25 (1995), no. 2, 739-756.

[19] Conditional Fourier-Feynman transforms and conditional convolution products, J. Korean Math. Soc. 38 (2001), no. 1, 61-76.

Seung Jun Chang

Department of Mathematics

DANKOOK UNIVERSITY

CheOnan 330-714, Korea

E-mail address: sejchang@dankook.ac.kr

JAE GIL CHOI

Department of Mathematics

DANKOOK UNIVERSITY

Cheonan 330-714, Korea

E-mail address: jgchoi@dankook.ac.kr

DAVID SKOUG

Department of Mathematics

UNIVERSITY OF NEBRASKA-LINCOLN

LINCOLN, NE 68588-0130, USA

E-mail address: dskoug@math.unl.edu 